Ueber Systeme von Gleichungen mit gewissen Besonderheiten.

Von

H. Krev in Freiburg i/Br.

Die Zahl der Lösungen eines Systems von $2 r$ Gleichnngen $f_{1}\left(x^{(1)} ; x^{(2)} ; \cdots ; x^{(r)}\right)=0, \cdots f_{2 r}\left(x^{(1)} ; x^{(2)} ; \cdots ; x^{(r)}\right)=0$,

deren linke Seiten ganze, $r$-fach ternäre Functionen der Coordinaten $x_{1}^{(h)}, x_{2}^{(h)}, x_{3}^{(h)}$ vour $r$ Punkten in der Ebene sind, und in den $x^{(h)}$ bezw. die Grade $n_{1}^{(h)}, \ldots n_{2 r}^{(h)}$ erreichen, ist bekanntlich im Allgemeinen durch den Ausdruck

$$
\sum_{1}^{2 r} n_{i}^{(1)} n_{k}^{(1)} n_{h}^{(2)} n_{l}^{(2)} \cdots n_{p}^{(r)} n_{q}^{\langle r\rangle}
$$

gegeben, in welchem die Indices $i, k, h, l, \ldots$ auf alle mögliche Arten verschiedene Zahlwerthe der Reihe $1,2, \ldots, 2 r$ annehnen. Derselbe gilt auch dann noch, wenn beliebig viele der Gleichungen nicht mehr alle $r$ Coordinatenreihen enthalten, die Zahlen $n$ also zum Theil Null sind.

In Folge besonderer Beschaffenheit der Gleichnngen kann, ohne dass zwischen ihnen eine Abhängigkeit bestände, die Zahl der Lösungen unendich gross werden. Es tritt dieses z. B. schon dann ein, wenn mehr als zwei der Functionen $f$ für $x^{(i)}=x^{(k)}$ identisch verschwinden; und allgemeiner könnte man die Annakme machen, dass $f_{h} \gamma_{h}^{(i, k)}$-fach verschwindet für $x^{(i)}=x^{(i)}, \vartheta_{h}^{(i)}$-fach für $x^{(i)}=a$, wo a einen festen Punkt (Ausnahmepunkt) der Ebene bedeutet. Wird in diesem Falle die beschränkende Bedingung hinzugefügt, dass $x^{(1)}, \cdots, x^{(r)}$ Gruppen von freien (d. h. nicht in Ausnahmepunkte fallenden), getrennt licgenden Punkten sein sollen, so bleibt die Zahl der Lösungen eine endliche, und es bietet sich die Aufgabe, die nicht nur von den $n$, sondern auch von den Zahlen $\gamma$, $\vartheta$ abhängige Reduction zu fiuden, welche der obige Ausdruck erfährt.

Zu den zahlreichen Specialfällen, welche diese Aufgabe in sich schliesst, gehört auch der aus nachstehender Annahme entspringende, 
der im Folgenden untersuche werden soll: Alle $\gamma$ and 9 haben den Werth 1, während die Gradzahlen $n$ sämmatlich von Null verschieden sind. Finen solchen tiall hat man vor sich, wenn z. B. jede Function $f$ aus Gliedern folgender Art sich zusammensetzt:

$$
\varphi \cdot\left|\begin{array}{cccc}
\psi_{1}\left(x^{(1)}\right) & \psi_{2}\left(x^{(1)}\right) & \cdots & \psi_{r}\left(x^{(1)}\right) \\
\psi_{1}\left(x^{(2)}\right) & \psi_{2}\left(x^{(2)}\right) & \cdots & \psi_{r}\left(x^{(2)}\right) \\
\cdot & \cdot & & \cdot \\
\cdot & \cdot & & \cdot \\
\psi_{1}\left(x^{(r)}\right) & \psi_{2}\left(x^{(r)}\right) & \cdots & \psi_{r}\left(x^{(r)}\right)
\end{array}\right|
$$

wo $\varphi$ eine beliebige Function bedeutet, und die Curven $\psi_{1}(x)=0, \cdots$, $\psi_{r}(x)=0$, welche von derselben Ordnung sind, einfach durch die Ausnahmepunkte gehen. Dieses Beispiel führt zugleich $z u$ einer weiteren Annahme, die hier gemacht werden soll, und die nicht nothwendig aus der ersten, hinsichtlich der Zahlen $\gamma$ und $\vartheta$ gemachten folgt, dass nämlich bei unendlicher Aunäherung eines der Punkte $x^{(2)}, \cdots x^{(r)}$ an einen Ausnahmepunkt, und beliebiger Lage der übrigen, die zugehörigen Curven $f_{h}\left(x^{(1)}\right)=0$, ohne auszuarten, die beiden benachbarten Punkte verbinden. - Es lässt sich zeigen, dass unter dicsen Voraussetzungen die Bestimmung der Anzahl brauchbarer Lösungen für jedes $r$ ausgeführt werden kann. Für $r=2$ und $r=3$ sind die fraglichen Ausdrücke im Folgenden entwickelt.

Zur Bestimmung von Punktegruppen im Raume sind $3 r$ Bedingungen

$$
f_{1}\left(x^{(1)} ; \cdots ; x^{(r)}\right)=0, \cdots, f_{3 r}\left(x^{(1)} ; \cdots ; x^{(r)}\right)=0
$$

erforderlich: auch hier erführt die im Allgemeinen gültige Zahl der Lösungen

$$
\sum_{l}^{3 r} n_{i}^{(1)} n_{k}^{(1)} n_{l}^{(1)} n_{l}^{(2)} n_{p}^{(2)} n_{q}^{(2)} \cdots n_{s}^{(r)} n_{t}^{(r)} n_{u}^{(r)}
$$

eine Reduction, wenn die Gleichungen die oben erwähnten Besonderheiten haben. -

\section{Abkürzende Bezeichnungen.}

Unter

$$
\left(\varphi_{1} \varphi_{2} \cdots \varphi_{2 r}\right)
$$

soll im Folgenden die Zahl der Punktgruppen in der Ebene verstanden werden, welche den $2 r$ Gleichungen $\varphi_{1}=0, \cdots, \varphi_{2 r}=0$ genügen. $g_{i}$ ist die Bedingung für $x^{(i)}$, auf einer gegebenen Geraden zu liegen; $g_{i k}$ die Bedingung für $x^{(i)}$ und $x^{(k)}$, in einer und derselben gegebenen Geraden zu liegen; $g_{i}^{2}$ sagt aus, dass der Punkt $x^{(i)}$ gegeben ist; $p_{i j k}$, dass die Verbindungsgerade von $x^{(i)}$ mit $x^{(k)}$ durch einen gegebenen Punkt geht; $p_{i k}^{2}$ ist gleichbedeutend mit $g_{i k}$. Hiernach ist z. B. (für $r=3$ ) die Bedeutung von 


\section{$\left(g_{1} p_{23} f_{1} f_{2} f_{3} f_{4}\right), \quad\left(g_{12} g_{3} f_{1} f_{2} f_{3}\right), \quad\left(g_{1}^{2} p_{12} p_{23} f_{1} f_{2}\right)$}

leicht ersichtlich.

Für Punktegruppen im Raume ist $g_{i k}^{*}$ die Bedingung, dass die Punkte $x^{(i)}, x^{(k)}$ ihren Verbindungsstrahl $v$ gegebene Gerade treffen lassen; $e_{i}, e_{i}{ }^{2}, e_{i}{ }^{3}$ sagt aus, dass $x^{(i)}$ in einer gegebenen Ebene, bezw. Geraden liege, oder endlich gegeben sei; also bedeutet z. B. (für $r=2$ )

$$
\left(e_{1} e_{2} g_{12} f_{1} f_{2} f_{3}\right)
$$

die Zahl der Punktepaare, welche $x^{(1)}, x^{(2)}$ in je eine von zwei gegebenen Ebenen werfen, die Verbindungsgerade eine einfache Bedingung èrfüllen lassen, und den Gleichungen $f_{1}=f_{2}=f_{3}=0$ gen ïgen.

Die Ordnung einer Curve, welche in Folge gegebener Bedingungen ein Punkt $x^{(i)}$ beschreibt, soll mit $\left(x^{(i)}\right)$ bezeichnet werden, ihre Vielfachheit in einem Ausnahmepunkte mit $v_{i}$; nur wenn sich der Punkt auf einer Geraden bewegt, ist die Bedeutung von $\left(x^{(i)}\right)$ und $v_{i}$ eine andere (vgl. den Hülfssatz).

Auf Gradzahlen beziggliche Summenausdrücke werden meistens nur durch ihr allgemeines Glied, mit Weglassung des Summenzeichens und der Indices, angedeutet, z. B. wird geschrieben

$$
\begin{aligned}
\mu \mu \nu & \text { für } \mu_{1} \mu_{2} \nu_{3}+\mu_{1} \mu_{3} \nu_{2}+\mu_{2} \mu_{3} \nu_{1}, \\
\mu \mu \nu \rho & \text { für } \sum_{1}^{4} \mu_{i} \mu_{k} \nu_{k} \varrho_{i}, \\
\mu \mu \nu \nu \varrho+\mu \mu & \text { für } \quad \sum_{1}^{5} \mu_{i} \mu_{k} \nu_{h} \mu_{l} \varrho_{m}+\sum_{1}^{5} \mu_{i} \mu_{k} ;
\end{aligned}
$$

die obere Grenze der Indices, welche nur verschiedene Werthe annehmen, ist jedesmal aus dem Zusammenhange ersichtlich. -

Die Zahl der Ausnahmepunkte wird im Folgenden immer mit $\alpha$ bezeichnet.

\section{$\S 1$.}

\section{Beweis eines Hülfssatzes.}

Sind die Punkte zweier Curven, eines $x$-Ortes und eines $y$-Ortes, eindeutig auf einander bezogen, und coincidirt an $c_{12}$ freien Stellen $x$ mit dem entsprechenden $y$, bestimmt ferner die Gleichung

$$
\varphi(x ; y)=0 \text {, }
$$

deren linke Seite für $x=y$ einfach verschwindet, für einen beliebig gegebenen Punkt y $\xi$ bewegliche Punkte $x$ des ersten Ortes, für einen beliebigen Punkt $x \boldsymbol{\eta}$ bewegliche Punkte $y$ des aweiten Ortes, dann giebt es

$$
\xi+\eta-c_{12}
$$

Mathematiouhe Annalen. XIX. 
Paare von zusammengehörigen Punkten $x, y$, welche der Gloichung $\varphi=0$ genïgen.

Der Beweis hierfür ergiebt sich sehr einfach durch Herstellung einer Correspondenz, die vermöge der Gleichung $\varphi\left(x^{x} ; y\right)=0$ jedem Punkte $x$ des ersten Ortes $\xi$ Punkte $x^{\prime}$, jedem $x^{\prime} \eta$ Punkte $x$ zuordnet. - Auf demselben Wege und mit Benutzung des schon erhaltenen Resultates zeigt man:

Sind die Punkte einer $x$ Curve, einer $y$-Curve und einer z-Curve eindeutig auf einander bezogen, bezeichnet $c_{12}, c_{13}, c_{23}$, wie oft an freier Stelle $x$ mit $y, x$ mit $z, y$ mit $z$ coincidirt, und bestimmt die Gleichung

$$
\varphi(x ; y ; z)=0,
$$

deren linke Scite für $x=y, x=z, y=z$ einfach verschwindet, für ein beliebiges Paar $x, y \xi$ bewegliche Punkte des $z$-Ortes, u. s. w., so ist

$$
\xi+\eta+\xi-c_{12}-c_{13}-c_{23}
$$

die Zahl der Tripel $x, y, z$, welche der Gleichung $\varphi=0$ genïgen.

Die Ausdehnung dieser Sätze auf Gruppen von beliebig vielen Curven giebt, wenn man noch die Zahlen $\xi, \eta, \xi, \ldots$ durch die Gradzahlen von $\varphi$, und durch die Ordnungen und Vielfachheiten der verschiedenen Oerter ausdrüekt, Folgendes:

Unter den $\infty^{1}$ Gruppen von Punkten $x^{(1)}, \cdots, x^{(r)}$, welche durch $2 r-1$ (bezw. $3 r-1$ ) Bedingungen bestimmt sind, gielt es

$$
n^{(1)}\left(x^{(1)}\right)+\cdots+n^{(r)}\left(x^{(r)}\right)-\sum v-\sum c_{i k},
$$

welche ciner Gleichung

$$
\varphi\left(x^{(1)} ; x^{(2)} ; \cdots ; x^{(r)}\right)=0
$$

genuigen, deren linke Seite für $x^{(i)}=x^{(k)}$ und fiur $x^{(i)}=a$ einfach verschwindet, und in den $x^{(i)}$ den Grad $n^{(i)}$ crreicht. $\Sigma v$ bedeutet die Summe der Viclfachheiten der $r$ Oerter in den Ausnahmepunten; $c_{i k}$ die Zahl der an freien Stellen stattfindenden Coincidenzen von $x^{(i)}$ mit $x^{(k)}$.

Verlangt eine der Bedingungen, dass sich $x^{(i)}$ auf einer gegebenen Geraden $g_{i}$ (bezw. $e_{i}^{2}$ ) bewege, so findet kein eindeutiges Entsprechen der Punkte mehr statt, vielmehr gehören zu einem beliebig auf $g_{i}$ gewählten Punkte im Allgemeinen mehrere Gruppen von $r-1$ Punkten. Der Satz bleibt aber auch in diesem Falle richtig, wenn man nur unter $\left(x^{(i)}\right)$ die Zahl der Gruppen versteht, welche ihren Punkt $x^{(i)}$ in einer beliebigen Geraden (bezw. Ebene) haben, and als den Antheil, welchen der $x^{(i)}$. Ort an $\Sigma v$ hat, nicht $\delta$, sondern $\delta\left(x^{(i)}\right)$ betrachtet, wo $\delta$ die Zahl der auf der Geraden liegenden Ausnahmepunkte bedeutet.

Bei beliebiger Annahme der Zahlen $\gamma$, für die Gleichung $\varphi=0$ würde an die Stelle von (1) der folgende Ausdruck treten:

$$
\xi+\eta-\gamma_{12} c_{12}-x \lambda \cdot \alpha
$$


hier giebt $x$ an, wie viele Coincidenzen von $x$ mit $y$ in jedem Ausnahmepunkte liegen; $\vartheta+\lambda$ ist die Vielfachheit der Grenzcurve $\varphi(x)=0$ in einem Punkte $a$, welche einem $z a$ a benachbarten $y$ entspricht. Wollte man also den Satz (I) verallgemeinern, so mïste man Zahlen cinführen, welche nicht von den $\gamma$ und $\vartheta$ alleine abhängen, sondern vielmehr von der Art, wie sich der $\vartheta^{(i)}$-fache Punkt der Curve $\varphi\left(x^{(i)}\right)=0$ in a und der $\gamma_{i k}$-fache Punzt in $x^{(k)}$ bei Annähenung von $x^{(k)}$ an a au einer neuen Singularität vereinigen. Dieser Umstand würde die Untersuchung des allgemeinen Falles erschweren. Ist aber $\lambda=0$, $\gamma=\vartheta=1$, so fallen jene Reductionen fort; man kaun dann immer den Satz (I) anwenden, mit alleiniger Ausnahme der Fälle, in welchen mehr als zwei Punkte gleichzeitig coineidiren.

\section{$\S 2$.}

Zurückführung der fraglichen Ausdrücke auf einfachere.

Der erste Schritt zur Ermittelung der Zahl

$$
\left(f_{1} f_{2} \cdots f_{2 r}\right)
$$

besteht in der Weglassung einer der Bedingungen, etwa $f_{2 r}$, und der Einführung der durch die übrigen Gleichungen definirten Curvengruppe. Auf die letztere wendet man den Sats (I) an, in welchem

$$
\left(x^{(i)}\right)=\left(g_{i} f_{1} \cdots f_{2 r-1}\right)
$$

zu setzen ist; $v_{i}$ ist nichts Anderes als die Verminderung, welche der Werth dieses Ausdrucks erfährt, wenn man die Gerade $g_{i}$, anstatt derselben eine beliebige Lage zu ertheilen, durch cinen Ausnahmepunit legt. Da sich ferner die $c_{i k}$ mit Hülfe des verallgemeinerten Chasles'schen Correspondenzprincips $\left.{ }^{*}\right)$ wie folgt bestimmen:

$$
c_{i k}=\left(x^{(i)}\right)+\left(x^{(i)}\right)-\left(p_{i k} f_{1} \cdots f_{2 r-1}\right),
$$

erhält man

$$
\begin{aligned}
\left(f_{1} f_{2}, \cdots f_{2 r}\right) & =\sum_{i=1}^{i=r}\left(n_{2 r}^{(i)}-r+1\right)\left(g_{i} f_{\mathrm{t}} \cdots f_{2 r-1}\right) \\
& +\sum_{i<k}\left(p_{i k} f_{1} \cdots f_{2 r-1}\right)-\alpha \sum v_{i}
\end{aligned}
$$

and dem analog für Punktegruppen im Raume:

$$
\begin{aligned}
\left(f_{1} f_{2} \cdots f_{3 r}\right) & =\sum_{i=1}^{i=r}\left(n_{3 r}^{(i)}-r+1\right)\left(e_{i} f_{1} \cdots f_{3 r-1}\right) \\
& +\sum_{i<k}\left(g_{i k} f_{1} \cdots f_{3 r-1}\right)-\alpha \sum v_{i} .
\end{aligned}
$$

*) Vgl. Sehubert: Kalkül d. abz. Geometrie pag. 44. 
Um die rechts stehenden noch unbekannten Ansdrücke weiter 20 reduciren, verfährt man genau wie es in der Theorie der Charakteristiken geschieht; man lässt wieder eine Bedingung $f$ aus, und sucht für die Schnar von Punktegruppen, welche durch die übrigen $\mathrm{Be-}$ dingungen gegeben ist, die Zahlen $(x), v, c$ zu bestimmen.

Ist ïberhaupt die Schaar der Gruppen definirt durch die Bedingungen

$$
g_{1} \cdots g_{k} g_{i k} g_{l, n} \cdots p_{k v} p_{\varrho \sigma} \cdots f_{1} \cdots f_{\lambda-1},
$$

so findet man

$$
\left(x^{(s)}\right)=\left(g_{1} \cdots g_{h} g_{s} g_{i k} g_{l m} \cdots p_{\mu \nu} p_{g \sigma} \cdots f_{1} \cdots f_{\lambda-1}\right) ;
$$

man wird also insbesondere auf Ausdrücke geführt, welche sich auf nur $r-1$ unbekannte Punkte beziehen, wenn $s$ unter den Indices der $g$ in (3) vorkommt; in diesem Falle ist $\mathrm{zu}$ beachten, dass der gegebene Punkt $x^{(s)}$ für die Gleichungen $f_{1}=0, \cdots f_{\lambda-1}=0$ als neuer Ausnahmepunkt auftritt. Ist die Bedingung $g_{s}$ nicht in (3) enthalten, so beschreibt $x^{(s)}$ eine Curve, deren Vielfachheit in den verschiedenen Ausnahmepunkten durch specielle Lagen der Geraden $g_{s}$ ermittelt wer. den kann.

Die $c$ bestimmen sich wieder (wenn nicht auf einfachere Art) durch das Correspondenzprincip, z. B.

$$
\begin{gathered}
c_{i k}=\left(x^{(i)}\right)+\left(x^{(k)}\right) ; \\
c_{\mu \nu}=\left(x^{(\mu i)}\right)+\left(x^{(v)}\right)-\left(g_{1} \cdots g_{k} g_{i k} g_{l m} \cdots g_{\mu \nu} p_{\varrho \sigma} \cdots f_{1} \cdots f_{\lambda-1}\right) ; \\
\text { u. s. f. }
\end{gathered}
$$

Durch Anwendung des Satzes (I) anf die Gruppenschaar (3) ergiebt sich also die Zurïchfiuhrung von

$$
\left(g_{1} \cdots g_{h} g_{i k} g_{l m} \cdots p_{\mu v} p_{\rho \sigma} \cdots f_{1} \cdots f_{h}\right)
$$

mif andere Zahlen, die sich entwoder auf nur $r-1$ unbekannte Punkte beziehen, oder einmal weniger $f$, cinmal melir die Bedingung g oder $p$ enthalten.

Da auch die auf Raumgruppen bezüglichen Zahlen eine ähnliche Reduction zulassen, so kommt man in allen Fällen schliesslich auf Systeme von elementaren Bedingungen, welche nur die Lage der Punkte in gegebenen Geraden oder Ebenen, und die Lage ihrer Verbindungsgeraden betreffen. Für die wirkliche Ausrechnung ist jedoch, wie die folgenden Beispiele zeigen, die Wegschaffung sämmtlicher Bedingungen $f$ nicht erforderlich.

$\$ 3$.

Punktepaare in der Ebene.

Die dreifachen Bedingungen
1) $g_{1} g_{2} f_{1}$
2) $g_{12} f_{1}$;
3) $g_{1} p_{12} f_{1}$
4) $g_{1} f_{1} f_{2}$
5) $p_{12} f_{1} f_{2}$

bestimmen Systeme von Punktepaaren, fur welche die Zahlen 


$$
(x),(y), c, \sum v
$$

zu berechnen sind. Die Gradzahlen der vier Gleichungen $f_{h}(x ; y)=0$ sollen mit $\mu_{h}, \nu_{k}$ bezeichnet werden; $\delta_{i}$ ist die Zahl der auf einer Geraden $g_{i}$ (oder $g_{i k}$ ) liegenden Ausnahmepunkte; $\delta_{i k}=1$ oder 0 , je nachdem der Schnittpunkt von $g_{i}, g_{k}$ Ausnahmepunkt ist oder nicht.

Für die Schaar 1) hat man die Zahlen

$$
\begin{gathered}
(x)=\nu_{1}-\delta_{2} ; \quad(y)=\mu_{1}-\delta_{1} ; \quad c=1-\delta_{12} ; \\
\sum v=\delta_{1}\left(v_{1}-\delta_{2}\right)+\delta_{2}\left(\mu_{1}-\delta_{1}\right)
\end{gathered}
$$

also folgt

$$
\begin{aligned}
\left(g_{1} g_{2} f_{1} f_{2}\right) & =\mu_{1} \nu_{2}+\mu_{2} \nu_{1}-1-\delta_{1}\left(\nu_{1}+\nu_{2}\right)-\delta_{2}\left(\mu_{1}+\mu_{2}\right) \\
& +2 \delta_{1} \delta_{2}+\delta_{12} .
\end{aligned}
$$

Ebenso leicht findet sich

$$
\begin{aligned}
\left(g_{12} f_{1} f_{2}\right) & =\left(\mu_{1}-1\right)\left(\nu_{2}-1\right)+\left(\mu_{3}-1\right)\left(\nu_{1}-1\right) \\
& -\delta_{1}\left(\mu_{1}+\nu_{1}+\mu_{2}+\nu_{2}-2 \delta_{1}-4\right) .
\end{aligned}
$$

In dem durch (3) definirten System ist

$$
\begin{gathered}
(x)=\nu_{1}-1 ;(y)=\mu_{1}+\nu_{1}-1-\delta_{1} ; \quad c=(y) ; \\
\sum v_{1}=\delta_{1}\left(\nu_{1}-1\right) ; \quad \sum v_{2}=\alpha-\delta_{1} ;
\end{gathered}
$$

letateres folgt aus dem Umstande, dass die $y$-Curve aturch alle Ausnahmepunkte, nur nicht darch die etwa auf $g_{1}$ gelegenen, einfach hindurchgeht. Hiernach wird

$$
\begin{aligned}
\left(g_{1} p_{12} f_{1} f_{2}\right) & =\left(\mu_{1}-1\right)\left(\nu_{2}-1\right)+\left(\mu_{2}-1\right)\left(\nu_{1}-1\right) \\
& +v_{1} \nu_{2}-1-\alpha-\delta_{1}\left(\nu_{1}+\nu_{2}-3\right)
\end{aligned}
$$

In dem System (4) ist

$$
(x)=\nu_{1} \nu_{z}-\alpha-1 ; \quad(y)=\mu_{1} \nu_{2}+\mu_{2} \nu_{1}-1-\delta_{1}\left(\nu_{1}+\nu_{2}\right) ;
$$

die Zahl (y) geht hervor aus (1) für $\delta_{2}=\delta_{12}=0$; derselbe Ausdruck lehrt, dass

$$
v_{2}=\mu_{1}+\mu_{2}-2 \delta_{1} \text { oder }=\mu_{1}+\mu_{1}-2 \delta_{1}-1
$$

je nachdem der Ausnahmepunkt beliebig oder auf $g_{1}$ gelegren ist, dass also

Feruer ist

$$
\sum v_{2}=\alpha\left(\mu_{1}+\mu_{2}-2 \delta_{1}\right)-\delta_{1} .
$$

$$
\begin{aligned}
c & =(x)+(y)-\left(g_{1} p_{12} f_{1} f_{2}\right) \\
& =\mu_{1}+\nu_{1}+\mu_{2}+\nu_{2}-3-3 \delta_{1} ;
\end{aligned}
$$

so folgt somit:

(4) $\left(g_{1} f_{1} f_{2} f_{3}\right)=\mu \nu v-v-(\alpha+1) \mu+3-\delta_{1}(\nu v-3 \alpha-5)$ 
Endlich erhält man für das durch (5) bestinmte Curvenpaar $(x)$ aus (3) für $\delta_{1}=0 ;(y)$ aus $(x)$ durch Vertauschung von $\mu$ mit $\nu$; $v_{1}$ ist der Coefficient von $-\delta_{1}$ in $(3)$, also

$$
\begin{gathered}
\sum v=\alpha\left(\mu_{1}+\mu_{2}+\nu_{1}+\nu_{2}-6\right) . \\
c=(x)+(y)-\left(g_{12} f_{1} f_{2}\right)_{\delta_{1}=0} \\
=\mu_{1} \mu_{2}+\nu_{1} \nu_{2}+\mu_{1} \nu_{2}+\mu_{2} \nu_{1}-\mu_{1}-\mu_{2}-\nu_{1}-\nu_{2}-2 \alpha .
\end{gathered}
$$

Man findet:

(5) $\left(p_{12} f_{1} f_{2} f_{3}\right)=\mu \mu \nu+\mu \nu \nu-\mu \mu-\nu \nu-\mu \nu-(\alpha-1)(\mu+\nu)+8 \alpha$. Hiermit sind alle zur Berechnung von $\left(f_{1} f_{2} f_{3} f_{4}\right)$ dienenden Zahlen bekannt; die Gleichung (1) \$2. giebt:

(b) $\left(f_{1} f_{2} f_{3} f_{4}\right)=\mu \mu \nu v-(\alpha+1)(\mu \mu+v v)-\mu \nu+3(\mu+v)$

$$
+6 \alpha^{2}+18 \alpha-6
$$

Insbesondere also erhält man für $\boldsymbol{\mu}_{h}=\nu_{h}=n$

$$
\left(f_{1} f_{2} f_{3} f_{4}\right)=6 n^{2}-12(\alpha+2) n^{2}+24 n+6 \alpha^{2}+18 \alpha-6 .
$$

Es soll hiervon eine Anwendung gemacht werden auf die Aufgabe, die $Z a h l$ der Punktepaare $x, y$ zu bestimmen, fuir welche alle zweireihigen Determinanten der Matrix

$$
\left\|\begin{array}{lllll}
\varphi_{1}(x) & \varphi_{2}(x) & \varphi_{3}(x) & \varphi_{4}(x) & \varphi_{5}(x) \\
\varphi_{1}(y) & \varphi_{2}(y) & \varphi_{3}(y) & \varphi_{4}(y) & \varphi_{5}(y)
\end{array}\right\|
$$

verschwinden; $\varphi_{1}=0, \cdots, \varphi_{5}=0$ sind die Gleichungen von 5 linear unabhängigen Curven $n^{t e r}$ Ordnung durch

feste Punkte.

$$
\alpha=\frac{n(n+3)}{2}-4
$$

Zur Abkürzung werde

$$
\varphi_{i}(x) \varphi_{k}(y)-\varphi_{k}(x) \varphi_{i}(y)=\Delta_{i k}
$$

gesetzt. Das System

$$
\Delta_{12}=\Delta_{23}=\Delta_{34}=\Delta_{45}=0
$$

hat nach (7)

$$
\frac{3}{2} n^{4}-9 n^{3}+\frac{45}{2} n^{2}-21 n+18
$$

Lösungen; unter ihnen sind unbrauchbare enthalten, welche den folgenden Systemen genügen

$$
\begin{aligned}
& \varphi_{2}(x)=\varphi_{2}(y)=\Delta_{34}=\Delta_{45}=0, \\
& \varphi_{3}(x)=\varphi_{3}(y)=\Delta_{12}=\Delta_{45}=0, \\
& \varphi_{4}(x)=\varphi_{4}(y)=\Delta_{12}=\Delta_{23}=0, \\
& \varphi_{2}(x)=\varphi_{4}(x)=\varphi_{2}(y)=\varphi_{4}(y)=0 .
\end{aligned}
$$


Jedes der drei ersten hat

$$
2\left(n^{2}-\alpha-1\right)^{2}-(n-1)(n-2)
$$

Lösungen, das System (13) dagegen

$$
\left(n^{2}-\alpha\right)\left(n^{2}-\alpha-1\right),
$$

die aber bereits in (10) und (12) vorkommen; es bleiben also

$$
\text { (9) }-3 \cdot(14)+(15)
$$

brauchbarc Lösungen des Systems (8), und da wegen der Symmetrie aller Gleichungen in $x, y$ von dieser Zahl die Hälfte zu nehmen ist, hat man den Satz:

$Z u \frac{n(n+3)}{2}-4$ willkülich in der Ebene gegebenen Punkten kann man aut

$$
\frac{1}{8}(n-2)(n-4)\left(n^{2}-9\right)
$$

Arten ein Punktepaar so bestimmen, dass durch die $\frac{n(n+3)}{2}-2$ Punlte noch eine dreifach unendliche Schaar von Curven n ${ }^{\text {ter }}$ Ordnumg geht.

Es möge hier noch, ohne Beweis, eine Verallgemeinerung der Gleichung (6) angegeben werden, welche für den Fall gilt, dass $f_{k}$ $\gamma_{h}$-fach verschwindet für $x=y, \vartheta_{h}$-fach für $x=a, \vartheta_{l}^{\prime}$-fach für $y=a$, und dass sich, bei dem Zusammenfallen von $y$ mit $a$, der $\vartheta_{l}$-fache und der $\gamma_{h}$-fache Punkt zu einem $\left(\vartheta_{h}+\gamma_{h}\right)$-fachen Punlite vereinigen:

$$
\begin{aligned}
\left(f_{1} f_{2} f_{3} f_{4}\right) & =\sum_{1}^{4} \mu_{i} \mu_{k} \nu_{l} \nu_{l}-\sum \gamma_{i} \gamma_{k}\left(\mu_{h}+v_{h}\right)\left(\mu_{l}+v_{l}\right) \\
& -\alpha \sum\left(\vartheta_{i} \vartheta_{k} v_{h} \nu_{l}+\mathfrak{\vartheta}_{i}^{\prime} \vartheta_{k}^{\prime} \mu_{h} \mu_{l}\right)+3 \sum \gamma_{i} \gamma_{k} \gamma_{h}\left(\mu_{l}+v_{l}\right) \\
& +\alpha^{2} \sum \vartheta_{i} \vartheta_{k} \vartheta_{h}^{\prime} \vartheta_{l}^{\prime}+\alpha \sum \gamma_{i} \gamma_{k}\left(\vartheta_{h} \vartheta_{l}+\vartheta_{h}^{\prime} \vartheta_{l}^{\prime}\right)-6 \gamma_{1} \gamma_{2} \gamma_{3} \gamma_{4} .
\end{aligned}
$$

Für $\gamma_{h}=\vartheta_{h}=\vartheta_{h}^{\prime}=1$ erhält man hieraus einen Ausdruck, der in dem numerischen Coefficienten von $\alpha$ von dem in (6) gefundenen abweicht.

$$
\S 4 .
$$

\section{Punktetripel in der Ebene.}

Die Grade der sechs Gleichungen $f_{h}(x ; y ; z)=0$ sollen mit $\mu_{h}$, $\nu_{h}, \varrho_{h}$ bezeichnet werden; $\delta_{i}$ und $\delta_{i k}$ haben wieder die im vorigen Paragraphen angegebene Bedeutung.

Zur Berechnung von $\left(f_{1} f_{2} f_{3} f_{1} f_{5} f_{6}\right)$ genügt es, die folgenden 11 Zahlen zu kennen: 
H. KrEY.
1) $\left(g_{1} g_{2} g_{3} f_{1} f_{2} f_{3}\right)$,
6) $\left(g_{12} p_{23} f_{1} f_{2} f_{3}\right)$,
2) $\left(g_{12} g_{3} f_{1} f_{2} f_{3}\right)$,
7) $\left(g_{1} p_{23} f_{1} f_{2} f_{3} f_{4}\right)$
3) $g_{123} f_{1} f_{2} f_{3}$,
8) $\left(g_{1} f_{1} f_{2} f_{3} f_{4} f_{5}\right)$
4) $\left.g_{1} g_{2} f_{1} f_{2} f_{3} f_{4}\right)$
9) $\left(g_{1} p_{12} p_{13} f_{1} f_{2} f_{3}\right)$
5) $\left.g_{12} f_{1} f_{2} f_{3} f_{4}\right)$
10) $\left(p_{12} p_{13} f_{1} f_{2} f_{3} f_{4}\right)$

11) $\left(p_{12} f_{1} f_{2} f_{3} f_{4} f_{5}\right)$

Jede derselben kann mit Hiilfe der vorangehenden und der in (1) -(6) § 3. gefundenen bestimmt werden, wie im Folgenden ausgeführt werden soll; die erforderlichen $Z$ wischenrechnungen werden dabei nur angedentet.

1) Schaar $g_{1} g_{2} g_{3} f_{1} f_{2}$.

Nach (1) \&3. ist

$$
\cdot(z)=\mu_{1} v_{2}+\mu_{2} v_{1}-1-\delta_{1}\left(v_{1}+\nu_{2}\right)-\delta_{2}\left(\mu_{1}+\mu_{2}\right)+2 \delta_{1} \delta_{2}+\delta_{12},
$$

und ähnliche Ausdrücke ergeben sich für $(y)$ und $(x)$. - Coincidenzen von $y$ mit $z$ können nur in dem Schnittpunkte von $g_{2}, g_{3}$ liegen, und auch nur dann, wenn dieser ein freier Punkt ist. Ersetzt man $\delta_{2}$ durch $\delta_{2}+1$, erhält man statt $(z)$ einen um $\mu_{1}+\mu_{2}-2 \delta_{1}$ kleineren Ausdruck, also ist

Da ferner

$$
c_{23}=\left(1-\delta_{23}\right)\left(\mu_{1}+\mu_{2}-2 \delta_{1}\right) \text {, u. s. w. }
$$

findet man

$$
\sum v=\delta_{1}(x)+\delta_{2}(y)+\delta_{3}(z)
$$

$$
\begin{aligned}
\left(g_{1} g_{2} g_{3} f_{1} f_{2} f_{3}\right) & =\mu \nu \rho-\mu-\nu-\rho-\delta_{1} \nu \rho-\delta_{2} \mu \rho-\delta_{3} \mu \nu \\
& +\left(2 \delta_{2} \delta_{3}+\delta_{23}\right) \mu+\left(2 \delta_{1} \delta_{3}+\delta_{13}\right) \nu+\left(2 \delta_{1} \delta_{2}+\delta_{12}\right) \varrho \\
& +3\left(\delta_{1}+\delta_{2}+\delta_{3}\right)-3\left(\delta_{1} \delta_{23}+\delta_{2} \delta_{13}+\delta_{3} \delta_{12}\right)-6 \delta_{1} \delta_{2} \delta_{3} .
\end{aligned}
$$

Treffen sich aber $g_{1}, g_{2}, g_{3}$, und awar in einem Ausnahmepunkte $A$, so ist der vorige Ausdruck zu ersetzen durch

$$
\begin{gathered}
\mu \nu \varrho-\delta_{1} \nu \varrho-\delta_{2} \mu \varrho-\delta_{3} \mu \nu+2 \delta_{2} \delta_{3} \mu+2 \delta_{1} \delta_{3} \nu+2 \delta_{1} \delta_{2} \varrho \\
-6 \delta_{1} \delta_{2} \delta_{3}-1
\end{gathered}
$$

Man hat nämlich hier einen der wenigen Fälle vor sich, in welchen mehr als zwei Punlte an derselben Stelle coincidiren. Von den

$$
\left(\mu_{1}-\delta_{1}\right)\left(\nu_{2}-\delta_{2}\right)+\left(\mu_{2}-\delta_{1}\right)\left(\nu_{1}-\delta_{2}\right)
$$

$z u z$ gehörenden Paaren $x, y$ geht eines verloren, wenn $z$ in $A$ fällt, und zwar dadurch, dass sowohl $x$ als $y$ sich dem Punkte $A$ nähert; die $z u$ einem solchen Paare gehörende ' $z^{\prime}$-Curve $f_{3}\left(x ; y ; z^{\prime}\right)=0$, welche eine Correspondenz zwischen Punkten $z$, $z^{\prime}$ auf $g_{3}$ vermittelt, artet nothwendig in eine Curve mit Doppelpuntet in $A$ aus; daher liegt eine unbrauchbare Coincidenz von $z^{\prime}$ mit $z$ in $A$. 
2) Schaar $g_{12} g_{3} f_{1} f_{2}$.

Hier ist (z) ohne Weiteres aus (2) \& 3. zu entnehmen; aus (1) $\$$ 3. findet man

$(x)=\nu_{1} \varrho_{2}+\nu_{2} \varrho_{1}-1-\left(1+\delta_{1}\right)\left(\varrho_{1}+\varrho_{2}\right)-\delta_{3}\left(\nu_{1}+\nu_{2}\right)+2 \delta_{3}\left(1+\delta_{1}\right)+\delta_{13} ;$

hieraus $(y)$ durch Vertauschung von $\mu$ und $\nu$. Rückt $z$ in den Schnittpunkt von $g_{12}, g_{3}$, ergiebt sich eine Verminderung des Ausdrucks (z). um

also ist

$$
\mu_{1}+\mu_{2}+\nu_{1}+\nu_{2}-6-4 \delta_{1}
$$

Da ferner

$$
c_{31}+c_{32}=\left(1-\delta_{13}\right)\left(\mu_{1}+\mu_{2}+\nu_{1}+v_{2}-6-4 \delta_{1}\right) \text {. }
$$

$$
c_{12}=(x)+(y) ; \quad \sum v=\delta_{1}(x)+\delta_{1}(y)+\delta_{3}(z),
$$

ergiebt sich

$$
\begin{aligned}
\left(g_{12} g_{3} f_{1} f_{2} f_{3}\right) & =\mu \nu \rho-\left(1+\delta_{1}\right)(\mu \varrho+v \varrho)-\delta_{3} \mu \nu \\
& +\left(\delta_{13}+2 \delta_{3}+2 \delta_{1} \delta_{3}-1\right)(\mu+\nu) \\
& +2\left(1+\delta_{1}\right)^{2}\left(\varrho-3 \delta_{3}\right)+\left(1-\delta_{13}\right)\left(8+6 \delta_{1}\right) .
\end{aligned}
$$

Hieran schiiesst sich die folgende, durch Induction leicht zu beweisende und auf Gruppen von beliebig vielen Punkten auszudehnende Gleichung

$$
\text { (3) } \begin{aligned}
\left(g_{123} f_{1} f_{2} f_{3}\right)= & \sum_{1}^{3}\left(\mu_{i}-2-\delta_{1}\right)\left(\nu_{k}-2-\delta_{1}\right)\left(\varrho_{h}-2-\delta_{1}\right) \\
= & \mu \nu \rho-\left(2+\delta_{1}\right)(\mu \nu+\mu \varrho+\nu \rho)+2\left(2+\delta_{1}\right)^{2}(\mu+\nu+\varrho) \\
& -6\left(2+\delta_{1}\right)^{3} .
\end{aligned}
$$

4) Schaar $g_{1} g_{2} f_{1} f_{2} f_{3}$.

Der Werth von $(z)$ geht hervor aus (1) für $\delta_{33}=\delta_{13}=\delta_{23}=0$, d. h. wenn der Geraden $g_{3}$ eine beliebige Lage ertheilt wird; $(x),(y)$ aus (4) §3. Die Vielfachheit der z-Curve in einem beliebigen Ausnahmepunkte ist

$$
v_{3}=\mu \nu-2 \delta_{2} \mu-2 \delta_{1} \nu-3+3 \delta_{12}+6 \delta_{1} \delta_{2}
$$

wie aus (1) für $\delta_{3}=1, \delta_{13}=\delta_{23}=0$ folgt; in jedem der $\delta_{1}-\delta_{12}$, bez. $\delta_{2}-\delta_{12}$ Punkte dagegen

$$
v_{3}-v+3 \delta_{2}, \text { bezw. } v_{3}-\mu+3 \delta_{1}
$$

endlich in dem etwaigen Ausnahmepunkte $\delta_{12}$ [vgl. (1 a)]

Hieraus

$$
v_{3}-\mu-v+3 d_{1}+3 \delta_{2}+1 \text {. }
$$

$$
\sum v_{3}=\alpha v_{3}-\delta_{1} v-\delta_{2} \mu+6 \delta_{1} \delta_{2}+\delta_{12} .
$$

Der $z$-Ort trifft $g_{1}$ an

$$
(z)-\delta_{1}\left(v_{3}-\nu+3 \delta_{2}\right)+\delta_{12}\left(\mu-3 \delta_{1}-1\right)
$$


freien Stellen; zieht man hiervon ab die Zahl $\left(g_{13} g_{2} f_{1} f_{2} f_{3}\right)$, die aus (2) durch Vertauschung von $\nu$ mit $\varrho, \delta_{3}$ mit $\delta_{2}, \delta_{13}$ mit $\delta_{12}$ hervorgeht, bleibt

$c_{13}=\mu \nu+\nu \rho-2 \delta_{2} \mu-2 \delta_{2} \varphi-\left(3+3 \delta_{1}\right) \nu-8+9 \delta_{2}+9 \delta_{1} \delta_{2}+7 \delta_{12}$. Hieraus $c_{23}$ durch Vertauschung von $\mu$ und $\nu, \delta_{1}$ mit $\delta_{2}$. Da endlich

so folgt

$$
c_{12}=\left(1-\delta_{12}\right)(\rho \rho-3 \alpha-8) \text {, }
$$

(4) $\left(g_{1} g_{2} f_{1} f_{2} f_{3} f_{4}\right)=\mu \nu \rho \varrho-\delta_{1} \nu \nu \rho-\delta_{2} \mu \mu \rho-(\alpha+2) \mu \nu-\mu \rho-v \varrho$

$$
\begin{aligned}
& +\left(2 \delta_{1} \delta_{2}+\delta_{12}-1\right) \varrho \varrho+\left(3+3 \delta_{2} \alpha+8 \delta_{2}\right) \mu \\
& +\left(3+3 \delta_{1} \alpha+8 \delta_{1}\right) \nu+3\left(\delta_{1}+\delta_{2}\right) \varrho+\left(6-12 \delta_{1} \delta_{2}-6 \delta_{12}\right) \alpha \\
& +24-12 \delta_{1}-12 \delta_{2}-40 \delta_{1} \delta_{2}-23 \delta_{12} .
\end{aligned}
$$

5) Schaar $g_{12} f_{1} f_{2} f_{3}$.

Aus (4) \& 3. erhält man $(y)$, wenn $\nu$ durch $\varrho, \alpha$ durch $\alpha+1$, $\delta_{1}$ durch $\delta_{1}+1$ ersetzt wird; die Gleichung (2) giebt Ordnung und Vielfachheit des $z$-Ortes, und zwar beträgt letztere in einem betiebigen. Ausnahmepurikte

$$
v_{3}=\mu \nu-\left(2+2 \delta_{1}\right)(\mu+\nu)+6\left(1+\delta_{1}\right)^{2},
$$

in einem auf $g_{12}$ liegenden jedoch nur

$$
v_{3}-\mu-\nu+8+6 \delta_{1} \text {. }
$$

Die Zahlen $c_{31}, c_{32}$ braucht man nicht einzeln zu kennen, sondern $n u r$ ihre Summe; diese ist

$$
c_{31}+c_{32}=(z)-\delta_{1}\left(v_{3}-\mu-\nu+8+6 \delta_{1}\right)-\left(g_{123} f_{1} f_{2} f_{3}\right) .
$$

Demnach wird

(5) $\left(g_{12} f_{1} f_{2} f_{3} f_{4}\right)=\mu \nu \rho \rho-\left(1+\delta_{1}\right)(\mu \rho \varrho+v \rho \varrho)-(\alpha+2) \mu \nu-\mu \rho-\nu \rho$

$$
\begin{aligned}
& -\left(2+4 \delta_{1}+2 \delta_{1}{ }^{2}\right) \rho+\left(11+3 \alpha+8 \delta_{1}+3 \delta_{1} \alpha\right)(\mu+\nu) \\
& +\left(8+6 \delta_{1}\right) \varrho-78-12\left(1+\delta_{1}\right)^{2} \alpha-110 \delta_{1}-40 \delta_{1}{ }^{2} .
\end{aligned}
$$

6) Schaar $g_{12} p_{23} f_{1} f_{2}$.

Aus (3) und (2) $\$ 3$. findet man

$(x)=\nu_{1} \varrho_{2}+\nu_{2} \varrho_{1}+\varrho_{1} \varrho_{2}-\nu_{1}-v_{2}-\left(2+\delta_{1}\right)\left(\varrho_{1}+\varrho_{2}\right)-\alpha+3+3 \delta_{1} ;$

$(y)=\mu_{1} \varrho_{2}+\mu_{2} \varrho_{1}-\mu_{1}-\mu_{2}-\left(1+\delta_{1}\right)\left(\varrho_{1}+\varrho_{2}\right)+2+2 \delta_{1}$.

Da auf einer durch $p_{23}$ gehenden Geraden $(y)$ Paare $y$, $z$ liegen, der feste Pundt sellst aber

$$
\left(\mu_{1}-1\right)\left(\nu_{2}-1\right)+\left(\mu_{2}-1\right)\left(\nu_{1}-1\right)-\delta_{1}\left(\mu_{1}+\mu_{2}+\nu_{1}+\nu_{2}-2 \delta_{1}-4\right)
$$

mal als Punkt $z$ auftritt, ist auch $(z)$ bekañnt; da ferner 


$$
\sum v_{3}=\left(\alpha-\delta_{1}\right)\left(\mu_{1}+\mu_{2}-2-2 \delta_{1}\right), \quad \sum c_{i k}=(x)+(y)+(z),
$$

rgiebt sich

6) $\left(g_{12} p_{23} f_{1} f_{2} f_{2}\right)=\mu \nu \varrho+\mu \mu \rho-\mu \nu-\left(2+\delta_{1}\right) \mu \varrho-\left(1+\delta_{1}\right)(\nu \rho+\varrho \varrho)$

$$
\begin{aligned}
& +\left(3-\alpha+3 \delta_{1}\right) \mu+2\left(1+\delta_{1}\right) \nu+\left(4+6 \delta_{1}+2 \delta_{1}^{2}\right) \varrho \\
& +3\left(1+\delta_{1}\right) \alpha-9-18 \delta_{1}-9 \delta_{1}^{2} .
\end{aligned}
$$

7) Schaar $g_{1} p_{23} f_{1} f_{2} f_{3}$.

Hier ergiebt sich $(x)$ aus (5) $\S 3$, wenn $\mu, v, \alpha$ durch $\nu, \varrho, \alpha+1$ zrsetzt werden. Die Vielfachheit des $z$-Ortes in $p_{23}$ beträgt

$$
\mu \nu \nu-\nu-(\alpha+2) \mu+3-\delta_{1}(\nu \nu-3 \alpha-8) \text { [vgl. (4) \& 3.], }
$$

während auf einer beliebigen Geraden durch $p_{23}$

$$
\left(g_{1} g_{23} f_{1} f_{2} f_{3}\right)_{\delta_{2}=0}
$$

Paare $y$, z liegen; $(z)$ ist also gleich der Summe dieser beiden Zahlen, deren zweite aus (2) durch Vertauschung von $\mu$ mit $\varrho, \delta_{3}$ mit $\delta_{1}, \delta_{1}$ mit $\delta_{2}$ hervorgeht. Der so entstehende Ausdruck giebt ausserlem als Summe der Vielfachheiten des $y$ - und des $z$-Ortes:

$$
v_{2}+v_{3}=\mu \nu+\mu \rho-2 \delta_{1}(\nu+\varrho)-6 \mu-6+18 \delta_{1}
$$

für einen beliebigen Ausnahmepunkt, dagegen

$$
v_{2}+v_{3}-\delta_{1}(y+\varphi-14)
$$

für einen auf $g_{1}$ liegenden; es ist mithin auch

$$
\sum\left(v_{2}+v_{3}\right)=\alpha\left(v_{2}+v_{3}\right)-\delta_{1}(\nu+\varrho-14)
$$

bekannt. - Die $y$ - und die $z$-Curve treffen $g_{1}$ zusammen an

$$
(y)+(z)-\delta_{1}\left[v_{2}+v_{3}-\delta_{1}(\nu+\rho-14)\right]
$$

freien Stellen; zieht man hierron ab die nach $(6)$ bekannten Zahlen

$$
\left(g_{12} p_{23} f_{1} f_{2} f_{3}\right), \quad\left(g_{13} p_{23} f_{1} f_{2} f_{3}\right)
$$

so bleibt $c_{21}+c_{31}$, während

ebenfalls bekannt ist. Man erhält

$$
c_{23}=(y)+(z)-\left(g_{1} g_{23} f_{1} f_{2} f_{3}\right)_{d_{2}=0}
$$

(7) $\left(g_{1} p_{23} f_{1} f_{2} f_{3} f_{4}\right)=\mu \nu \rho \varrho+\mu \nu \nu \rho-\mu \nu \nu-\mu \varrho \varrho-\mu \nu \rho-\delta_{1}(\nu \nu \rho+\nu \varrho \varrho)$

$$
\begin{aligned}
& -\alpha(\mu \nu+\mu \rho)+\left(2 \delta_{1}-1\right)(v \nu+\varrho \varrho)+\left(2 \delta_{1}-2\right) \nu \varrho \\
& +\left(11+2 \delta_{1}+3 \delta_{1} \alpha\right)(\nu+\varrho)+8(\alpha+1) \mu \\
& +\left(12-32 \delta_{1}\right) \alpha-54-64 \delta_{1} .
\end{aligned}
$$

8) Schaar $\left(g_{1} f_{1} f_{2} f_{3} f_{4}\right)$.

Zu einem auf $g$ gegebenen Punkte $x$ gehören so viele Paare $y, z$, wie der Ausdruck (6) \& 3. angiebt, in welchem $\mu$ durch $\varrho$, 
$\alpha$ durch $a+1$ zu ersetzen ist. Die Gleichung (4) liefert ausser dem Werthe von $(y)$ noch

wo

$$
\sum v_{2}=\alpha v_{2}-\delta_{1}(\varrho \varrho-6 \alpha-23)
$$

$v_{2}=\mu \varrho \varrho-2 \delta_{1} \varrho \varrho-(3 \alpha+8) \mu-3 \varrho+12+12 \delta_{1} \alpha+40 \delta_{1}$.

Man hat weiter

$$
c_{23}=(y)+(z)-\left(g_{1} p_{29} f_{1} f_{2} f_{3} f_{1}\right)
$$

was nach (7) bekannt ist;

$$
c_{12}=(y)-\delta_{1}\left(v_{2}-\varrho \varrho+6 \alpha+23\right)-\left(g_{12} f_{1} f_{2} f_{3} f_{4}\right),
$$

was sich ebenfalls nach (5) berechnen lässt. Aus $(y), \Sigma v_{2}, c_{12}$ entsteht $(z), \Sigma v_{3}, c_{13}$ durch Vertauschung von $\nu$ mit $\varrho .-$ Es wird hiernach

$$
\begin{aligned}
\left(g_{1} f_{1} f_{2} f_{3} f_{4} f_{5}\right) & =\mu \nu \nu \rho \varrho-\delta_{1} \cdot \nu \nu \rho \varrho-\left(\alpha+\delta_{1}\right)(\mu \nu \nu+\mu \rho \varrho) \\
& -\mu \nu \rho-\nu \nu \rho-\nu \rho \varrho+3 \mu \nu+3 \mu \rho \\
& +\left(3+8 \delta_{1}+3 \delta_{1} \alpha\right)(\nu \nu+\varrho \varrho)+3 \delta_{1} \cdot v \varrho \\
& +\left(6 \alpha^{2}+30 \alpha+18\right) \mu+\left(6 \alpha+24-12 \delta_{1}\right)(\nu+\varrho) \\
& -306-\delta_{1}\left(30 \alpha^{2}+190 \alpha+230\right) .
\end{aligned}
$$

Diese Gleichung lehrt sowohl die Ordnung des durch

$$
f_{1}=f_{2}=\cdots=f_{5}=0
$$

bestimmten $(x)$-Ortes, als die Vielfachheit desselben in den Ausnahmepunkten kenuen.

9) Schaar $g_{1} p_{12} p_{13} f_{1} f_{2}$.

Einem gegebenen $(x)$ entsprechen

$$
(x)=\left(\nu_{1}-1\right)\left(\rho_{2}-1\right)+\left(\nu_{2}-1\right)\left(\varrho_{1}-1\right)
$$

Paare $y, z$. Die Vielfachheit des $z$-Ortes in $p_{13}$ beträgt

$$
\left(g_{3}^{2} g_{1} p_{12} f_{1} f_{2}\right)
$$

was nach $(3) \S 3$. berechnet werden kann; demuach ist

$$
(z)=(x)+\left(g_{3}^{2} g_{1} p_{12} f_{1} f_{2}\right) ; \quad(y)=(x)+\left(g_{2}^{2} g_{1} p_{13} f_{1} f_{2}\right)
$$

Liegt $x$ in dem Schuittpunkte von $g$, wit der Verbindungsgeraden $p_{12} p_{13}$, so giebt es auf dieser Geraden nur noch

$$
\left(\nu_{1}-2\right)\left(\varphi_{2}-2\right)+\left(\nu_{2}-2\right)\left(\varphi_{1}-2\right)
$$

Paare von getrennt liegendeu Punkten $y, z$; und da jedes verloren gehende Paar eine Coincidenz von y mit $z$ anzeigt, hat man

$$
c_{23}=\nu_{1}+\nu_{2}+\varrho_{1}+e_{2}-6 \text {. }
$$


Fudlich ist

$$
\begin{array}{cl}
c_{13}=(z), & c_{23}=(y), \\
\sum v_{3}=\left(\alpha-\delta_{1}\right)\left(\nu_{1}+\nu_{2}-2\right) ; \quad \sum v_{2}=\left(\alpha-\delta_{1}\right)\left(\rho_{1}+\rho_{2}-2\right) .
\end{array}
$$

Hicraus:

$$
\begin{aligned}
\left(g_{1} p_{12} p_{13} f_{1} f_{2} f_{3}\right) & =\mu \nu \rho+\nu \nu \rho+\nu \rho \varrho-v \nu-\rho \varrho-2 \nu \rho-\mu \nu \\
& -\mu \varrho+2 \mu-\delta_{1} \nu \rho+\left(2+3 \delta_{1}-\alpha\right)(\nu+\varrho) \\
& +6 \alpha+2-12 \delta_{1} .
\end{aligned}
$$

\section{0) Schaar $p_{12} p_{13} f_{1} f_{2} f_{3}$}

Nach (9) sind bekannt $(x)$ und

$$
v_{1}=\nu \varrho-3 \nu-3 \varrho+12 .
$$

Die Vielfachheit der $y$-Curve in $p_{12}$ ist

man hat also

$$
\left(g_{2}^{2} p_{13} f_{1} f_{2} f_{3}\right) \quad[\mathrm{vgl} .(5) \$ 3 .]
$$

$$
\begin{aligned}
& (y)=\left(g_{2}^{2} p_{13} f_{1} f_{2} f_{3}\right)+\left(g_{12} p_{13} f_{1} f_{2} f_{3}\right)_{\delta_{1}=0}, \quad[\text { vgl. }(6)], \\
& (z)=\left(g_{3}^{2} p_{12} f_{1} f_{2} f_{3}\right)+\left(g_{13} p_{12} f_{1} f_{2} f_{3}\right)_{\delta_{1}=0} .
\end{aligned}
$$

Der Coefficient von $-\delta_{1}$ in (6) giebt, nach Vertauschung von $\mu$ mit $\nu$,

$$
v_{1}+v_{2}=v \rho+\mu \varphi+\varphi \varrho-2 \mu-3 v-8 \rho-3 \alpha+27,
$$

und da $v_{1}$ bereits gefunden ist:

$$
\begin{aligned}
& v_{2}=\mu \rho+\varrho \varrho-5 \rho-2 \mu-3 \alpha+15 \\
& v_{3}=\mu \nu+\nu \nu-5 \nu-2 \mu-3 \alpha+15 .
\end{aligned}
$$

Die $c_{12}, c_{13}$ bestimmen sich mit Hülfe von (6). - Coincidenzen von $y$ mit $z$ können nur auf der Geraden $p_{12} p_{13}$ liegen, welche letztere von dem $x$-Orte, ansser in den beiden festen Punkten, noch in einer bekannten Anxahl von Stellen getroffen wird. Zu diesen gehören jedenfalls die in den

$$
\left(g_{123} f_{1} f_{2} f_{3}\right)=\sum_{1}^{3}\left(\mu_{i}-2\right)\left(v_{k}-2\right)\left(\varrho_{h}-2\right)
$$

Tripelı getrennt liegender Punkte enthaltenen $x$; ausserdem aber

$$
c_{23}=\mu \nu+\mu \varrho-6 \mu-3 \nu-3 \varrho+28
$$

andere, deren zugehörige $y$, z zusammenfallen. - Das Ergebniss ist:

$$
\begin{aligned}
& \left(p_{12} p_{13} f_{1} f_{2} f_{3} f_{4}\right)=\mu \mu \nu \rho+\mu \nu \nu \rho+\mu \nu \rho \varrho+\nu \nu \rho \varrho-\mu \nu \nu-\mu \rho \varrho \\
& -2 \mu \nu \rho-\mu \mu \nu-\mu \mu \rho+2 \mu \mu+2 \mu \nu+2 \mu-\nu-\varrho+2 \mu \rho \\
& -2 \nu \rho \varrho-2 \nu \nu \rho+4 \nu \rho+3 \nu \nu+3 \varrho \rho-\alpha \mu \nu-\alpha \mu \rho-\alpha \nu \nu \\
& -\alpha \nu \rho-\alpha \rho \varrho+6 \alpha \mu+11 \alpha(\nu+\varrho)+6 \alpha^{2}-70 \alpha-48 .
\end{aligned}
$$




\section{1) Schaar $p_{12} f_{1} f_{2} f_{3} f_{4}$.}

Die Mittel zur Berechnung der charakteristischen Zahlen für dieses Curventripel sind im Vorhergelienden enthalten.

Nach Vertauschung von $\mu$ mit $\varrho, \delta_{1}$ mit $\delta_{3,}$ in (7) erhält man $(z)$ und $v_{3}$. Die Zahlen $(x),(y)$ bestimmt man wieder am bequemsten mit Hülfe des Princips der speciellen Lage, z. B.

$$
(x)=\left(g_{1}^{2} f_{1} f_{2} f_{3} f_{1}\right)+\left(g_{12} f_{3} f_{2} f_{3} f_{4}\right)_{J_{1}=0} .
$$

Fermer hat man:

$$
\begin{aligned}
& c_{12}=\left(g_{1}^{2} f_{1} f_{2} f_{3} f_{4}\right)+\left(g_{2}{ }^{2} f_{1} f_{2} f_{3} f_{4}\right)+\left(g_{12} f_{1} f_{2} f_{3} f_{4}\right)_{\delta_{1}=0} \\
& c_{13}=(x)+(z)-\left(p_{12} p_{13} f_{1} f_{2} f_{3} f_{4}\right)
\end{aligned}
$$

endlich geben die in (5) mit $-\delta_{1}$ und ${ }^{\circ}-\delta_{1}{ }^{2}$ multiplicirten Glieder den Werth von $v_{1}+v_{2}$. Man findet

$$
\begin{aligned}
& \left(p_{12} f_{1} f_{2} f_{3} f_{4} f_{5}\right)=\mu \nu \nu \rho \varrho+\mu \mu \nu \rho \varrho-\nu \nu \rho \varrho-\mu \mu \rho \varrho-\mu \nu \rho \varrho \\
& -\alpha(\mu \nu \nu+\mu \varrho \varrho+\nu \rho \varrho+\mu \mu \nu)-2 \mu \mu \nu-2 \mu \nu \nu-2 \mu \nu \rho \\
& -\mu \mu \rho-\nu \nu \rho+11(\mu \varrho+\nu \rho)+14 \mu \nu+3 \alpha \mu \nu+8(\alpha+1) \varrho \varrho \\
& +(3 \alpha+11)(\mu \mu+\nu \nu)+(12 \alpha-54) \varrho+\left(6 \alpha^{2}+18 \alpha-60\right)(\mu+\nu) \\
& -80 \alpha^{2}-462 \alpha+174 .
\end{aligned}
$$

Die Zahlen (8) und (11) dienen żur Bestimmung des gesuchten Ausdrucks; mit Hiilfe von (1) \& 2. wird

$$
\begin{aligned}
& \left(f_{1} f_{2} f_{3} f_{4} f_{5} f_{6}\right)=\mu \mu \nu \nu \rho \varrho-\mu \mu \nu \rho-\mu \nu v \rho-\mu \nu \rho \varrho \\
& +3(\mu \mu \nu+\mu \nu \nu+\mu \mu \rho+\mu \rho \varrho+\nu \nu \rho+\nu \rho \varrho) \\
& -(\alpha+2)(\mu \mu \nu \nu+\mu \mu \rho \rho+\nu \nu \rho \varrho) \\
& +(6 \alpha+24)(\mu \nu+\mu \varrho+\nu \rho)+\left(6 \alpha^{2}+30 \alpha+18\right)(\mu \mu+\nu \nu+\varrho \varrho) \\
& -(60 \alpha+306)(\mu+\nu+\varrho)-90 \alpha^{3}-810 \alpha^{2}-1716 \alpha+2358 .
\end{aligned}
$$

Insbesondere ist also bei Gleichheit aller Gradzahlen und Symmetrie der Gleichungen in $x, y, z$ die Zahl der Lösungen

(13) $15 n^{6}-(360+90 \alpha) n^{4}+360 n^{3}+\left(90 \alpha^{2}+630 \alpha+990\right) n^{2}$

$-(360 \alpha+1836) n-15 \alpha^{3}-135 \alpha^{2}-286 \alpha+393$.

Es sei bemerkt, dass die Gleichung (12) [und dasselbe gilt von (6) § 3.] nicht mehr in Anspruch genommen werden darf, wenn einige der Gradzahlen Null sind. Dieses ist in der Herleitung des Satzes (I) begründet. Enthielte die in $\$ 1$. mit $\varphi$ bezeichnete Function nicht die Coordinaten aller $r$ Punkte, sondern nur $x^{(1)}, \ldots$, $x^{(s)}$ (wo $s<r$ ), so würde an die Stelle von (I) folgender Ausdruck treten:

$$
n^{(1)}\left(x^{(1)}\right)+\cdots+n^{(s)}\left(x^{(s)}\right)-\sum\left(v_{1}+\cdots+v_{s}\right)-\sum_{i}^{s} c_{i k},
$$


der sich nicht durch die Specialisirung $n^{(s+1)}=\cdots=n^{(r)}=0$ ans dem allgemeinen herleiten lässt. Fehlt z, B. $z$ in $f_{5}$, so braucht man von den auf die Schaar 8) bezüglichen Kahlen nur $(x),(y), v_{1}, v_{2}, c_{12}$ zu kennen, um die Berechnung von $\left(g_{1} f_{1} f_{2} f_{3} f_{4} f_{5}\right)$ auszuführen. - Im Uebrigen bleibt die Rechnung genau dieselbe, nur muss sie für derartige Fialle besonders wiederholt werden.

\section{$\S 5$.}

\section{Punktepaare im Raume.}

Im Folgenden bezeichnet $\delta_{i}, \delta_{i i}, \delta_{i k}$ die Zahl der Ausnahmepunkte, die bzw. in der Ebene $e_{i}$, der Geraden $e_{i}{ }^{2}$, der Geraden $e_{i} e_{k}$ liegen; $\delta_{i i k}$ ist $=1$ oder 0 , je nachdem der Punkt $e_{i}^{2} e_{k}$ Ausuahmepunkt ist oder nicht.

Ohne weitere Rechnung bekannt sind die nachstehenden Zahlen, welche den später zu entwickelnden zu Grunde gelegt werden:

a) $\left(e_{1}^{3} g_{12}{ }^{2} f_{1}\right)=\nu_{1}-1 ; \quad$ b) $\left(e_{1}^{3} e_{2} g_{12} f_{1}\right)=\nu_{1} ; \quad$ c) $\left(e_{1}^{2} g_{12} f_{1}\right)=2 \nu_{1}-2$.
d) $\left(e_{1}{ }^{2} e_{2} g_{12}{ }^{2} f_{1}\right)=\mu_{1}+2 \nu_{1}-1-\delta_{11}$;
e) $\left(e_{1}^{2} e_{2}^{2} g_{12} f_{1}\right)=\mu_{1}+\nu_{1}-\delta_{11}-\delta_{22}$;

f) $\left(e_{1} e_{2} g_{12}^{3} f_{1}\right)=2 \mu_{1}+2 v_{1}-2$

g) $\left(e_{1} g_{12}{ }^{4} f_{1}\right)=2 \nu_{1}-2$

h) $\left(e_{1}^{3} e_{2} f_{1} f_{2}\right)=\nu_{1} v_{2}-\delta_{2}$

i) $\left(e_{1}^{3} g_{12} f_{1} f_{2}\right)=\nu_{1} \nu_{2}-1$;

k) $\left(g_{12}^{4} f_{1} f_{2}\right)=2\left(\mu_{1}-1\right)\left(v_{2}-1\right)+2\left(\mu_{2}-1\right)\left(\nu_{1}-1\right)$;

l) $\left(e_{1}^{*} e_{2}^{2} f_{1} f_{2}\right)=\left(\mu_{1}-\delta_{11}\right)\left(\nu_{2}-\delta_{22}\right)+\left(\mu_{2}-\delta_{11}\right)\left(\nu_{1}-\delta_{22}\right)$.

Die Gleichungen e) und l) gelten im Allgemeinen richt mehr, wenn sich die Geraden $e_{1}{ }^{2}, e_{2}{ }^{2}$ treffen; wohl aber - und dieser Fall kommt allein in Betracht - wenn der Schnittpunkt Ausnahmepunkt ist.

Da die Berechnung der noch erforderlichen 16 Zahlen, welche wieder durch Abtrennung einer Bedingung $f$ und Einfuihrung einer Schaar von Punltepaaren geschieht, in allen Fällen leicht ist, sollen nur die Resultate und die zur Herleitung benutzten Zahlen angegeben werden.

$$
\begin{gathered}
\left(e_{1}^{2} g_{12}{ }^{2} f_{1} f_{2}\right)=\mu_{1} \nu_{2}+\mu_{2} \nu_{1}-\mu_{1}-\mu_{2}-\nu_{1}-\nu_{2}+2 \nu_{1} \nu_{1} \\
-\delta_{11}\left(\nu_{1}+\nu_{2}-2\right) \\
\text { aus a), c) und d). }
\end{gathered}
$$

$$
\begin{gathered}
\left(e_{1}^{2} e_{2} g_{12} f_{1} f_{2}\right)=\mu_{1} v_{2}+\mu_{2} \nu_{1}+\nu_{1} \nu_{2}-1-\delta_{11}\left(\nu_{1}+\nu_{2}\right)-\delta_{2}+\delta_{112} \\
\text { aus b) und e }), \text { wegen } c=1-\delta_{112} . \\
\left(e_{1} g_{12}^{3} f_{1} f_{2}\right)=2\left(\mu_{1} \nu_{2}+\mu_{2} \nu_{1}+\nu_{1} \nu_{2}-\mu_{1}-\mu_{2}-v_{1}-\nu_{2}+1\right) \\
\text { ans c }), f) \text { und } \mathrm{g}) .
\end{gathered}
$$


(4) $\left(e_{1} e_{2} g_{12}{ }^{2} f_{1} f_{2}\right)=2\left(\mu_{1} \nu_{2}+\mu_{2} \nu_{1}\right)+\mu_{1} \mu_{2}+\nu_{1} \nu_{2}-\mu_{1}-\mu_{2}-\nu_{1}$ $-\nu_{2}-\delta_{1}-\delta_{2}+\delta_{12}$ aus d) und f), wegen $e=\mu_{1}+\nu_{1}-\delta_{12}$.

(5) $\left(e_{1}^{2} g_{12} f_{1} f_{2} f_{3}\right)=\mu \nu \nu+\nu_{1} \nu_{2} \nu_{3}-\delta_{11}(\nu \nu-4)-\mu-\nu-\alpha+2$ aus i), (1) und (2).

(6) $\left(e_{1} g_{12}{ }^{2} f_{1} f_{2} f_{3}\right)=\mu \mu \nu+\nu_{1} \nu_{2} \nu_{3}+2 \mu \nu \nu-\mu \mu-\nu \nu-\mu \nu-\alpha$ aus (1), (3), (4).

(7) $\left(e_{1} e_{2} g_{12} f_{1} f_{2} f_{3}\right)=\mu \mu \nu+\mu \nu \nu-\mu-\nu-\delta_{1} \nu-\delta_{2} \mu+2+4 \delta_{12}$ aus (2) und (4).

(8) $\quad\left(g_{12}{ }^{3} f_{1} f_{2} f_{3}\right)=2(\mu \mu \nu+\mu \nu \nu-\mu \mu-\nu \nu-\mu \nu+\mu+\nu)$ aus (k) und (3).

(9) $\left(e_{1} g_{12} f_{1} f_{2} f_{3} f_{4}\right)=\mu \mu \nu \nu+\mu \nu \nu \nu-\mu \mu-\nu \nu-\mu \nu+2 \mu+2 \nu$ $-\alpha \mu-2-\delta_{1}(\nu \nu-12)$ aus $(5),(6),(7)$.

(10) $\left(g_{12}^{2} f_{1} f_{2} f_{3} f_{4}\right)=\mu \mu \mu \nu+\mu \nu \nu \nu+2 \mu \mu \nu \nu-\mu \mu \mu-\nu \nu \nu-\mu \mu \nu$ $-\mu \nu \nu+2 \mu+2 \nu-\alpha(\mu+\nu-10)-4$ aus $(6)$ und (8).

(11) $\left(g_{12} f_{1} f_{2} f_{3} f_{4} f_{5}\right)=\mu \mu \mu \nu \nu+\mu \mu \nu \nu \nu-\mu \mu \mu-\nu \nu \nu-\mu \nu \nu$ $-\mu \mu \nu+2 \mu \mu+2 \nu \nu+2 \mu \nu-2 \mu-2 \nu-\alpha(\mu \mu+\nu \nu-34)$ aus (9) und (10).

(12) $\left(e_{1}^{2} e_{2} f_{1} f_{2} f_{3}\right)=\mu \nu \nu-\delta_{11} \nu \nu-\delta_{2} \mu+3 \delta_{11} \delta_{2}-1+\delta_{112}$ aus $h$ ), l) und (2).

(13) $\left(e_{1}^{2} f_{1} f_{2} f_{3} f_{4}\right)=\mu \nu \nu \nu-\mu-\nu-\alpha \mu+4-\delta_{11}(\nu \nu \nu-4 \alpha-6)$ aus (12) und (5), wegen $(x)=\nu_{1} \nu_{2} \nu_{3}-\alpha-1$.

(14) $\quad\left(e_{1} e_{2} f_{1} f_{2} f_{3} f_{4}\right)=\mu \mu \nu \nu-\mu-\nu+4-\delta_{1} \nu \nu-\delta_{2} \mu \mu$ aus (7) und (12).

$$
+6 \delta_{1} \delta_{2}+6 \delta_{12}
$$

(15) $\left(e_{1} g_{12} f_{1} f_{2} f_{3} f_{1}\right)=\mu \mu \nu \nu+\mu \nu \nu \nu-\mu \mu-\nu \nu-\mu \nu-\alpha \mu$ $-\delta_{1} \nu \nu+2 \mu+2 \nu-2+12 \delta_{1}$ aus (5), (6), (7).

(16) $\left(e_{1} f_{1} f_{2} f_{3} f_{4} f_{\mathrm{s}}\right)=\mu \mu \nu \nu \nu-\delta_{1} \nu \nu \nu-(\alpha+1) \mu \mu-\nu \nu-\mu \nu$ $+4 \mu+4 \nu-10+10 \delta_{1} \alpha+24 \delta_{1}$ aus $(13),(14),(15)$. 
Die Zahlen (11) und (16) sind es, aus welchen, nach (2) § 2., die gesuchte sich unmittelbar zusammensetzt. Man findet:

$$
\begin{aligned}
\left(f_{1} f_{2} f_{3} f_{4} f_{5} f_{6}\right) & =\mu \mu \mu \nu \nu \nu-(\alpha+1)(\mu \mu \mu+\nu \nu \nu)-\mu \mu \nu-\mu \nu \nu \\
& +4(\mu \mu+\nu \nu+\mu \nu)-10(\mu+\nu) \\
& +20 \alpha^{2}+82 \alpha+20 .
\end{aligned}
$$

$\S 6$.

Punktegruppen auf einer Fläche.

Die Aufgabe, Punktegruppen auf einer gegebenen Fläche $\boldsymbol{F}=0$ zu finden, welche $2 r$ Bedingungen erfüllen, ist einerseits ein specieller Fall der auf Gruppen im Raume bezïglichen Probleme, sofern $r$ Bedinguugen nur aussagen, dass jeder Punkt auf $F$ liegen soll; andererseits eine Verallgemeinerung der für Punltegruppen in der Ebene zu stellenden, da eine Gleichung

$$
f_{h}\left(x^{(1)} ; \cdots ; x^{(r)}\right)=0
$$

in Verbindung mit $F=0,2(r-1)$-fach unendliche Systeme von Curven auf $F$ darstellt, die in den Ausnahmepunkten und für $x^{(i)}=x^{(k)}$ das bekannte Verhalten zeigen.

Die Berechnung der fraglichen Zahlen ist nicht schwieriger als die für Gruppen in der Ebene, wie an dem Beispiele $r=2$ kurz ausgeführt werden möge. $F$ sei (als Punlitfläche) ohne Singularitäten, und von der Ordnung $m$.

Von den bekannten Zahlen

$$
\begin{aligned}
& \left(e_{1}^{2} e_{2} g_{12}\right)=m^{2}, \quad\left(e_{1} e_{2} g_{12}{ }^{2}\right)=2 m^{2}-m, \\
& \left(e_{1}^{2} e_{2} f_{1}\right)=\left(m-\delta_{11}\right)\left(m v_{1}-\delta_{2}\right) ; \quad\left(e_{1}^{2} f_{1} f_{2}\right)=\left(m-\delta_{11}\right)\left(m v_{1} v_{2}-\alpha-1\right)
\end{aligned}
$$

ausgehend, berechnet man leicht der Reihe nach die folgenden:

(1) $\left(e_{1} e_{2} g_{12} f_{1}\right)=m^{2}\left(\mu_{1}+\nu_{1}\right)-m\left(1+\delta_{1}+\delta_{2}\right)+\delta_{12}$.

(2) $\quad\left(e_{1} e_{2} f_{1} f_{2}\right)=m^{2}\left(\mu_{1} \nu_{2}+\mu_{2} \nu_{1}\right)-m\left[1+\delta_{1}\left(\nu_{1}+v_{2}\right)+\delta_{2}\left(\mu_{1}+\mu_{2}\right)\right]$

$$
+2 \delta_{1} \delta_{2}+\delta_{12} \text {. }
$$

(3) $\left(e_{1} g_{12} f_{1} f_{2}\right)=m^{2}\left(\mu_{1} \nu_{2}+\mu_{2} \nu_{1}+\nu_{1} \nu_{2}\right)-m^{2}-m\left(\mu_{1}+\mu_{2}+\nu_{1}+v_{2}\right)$

$$
-m(\alpha-2)-\delta_{1}\left(m \nu_{1}+m \nu_{2}-3\right) \text {. }
$$

$$
\begin{aligned}
\left(g_{12}{ }^{2} f_{1} f_{2}\right) & =m^{2}\left(\mu_{1} \mu_{2}+\nu_{1} \nu_{2}+2 \mu_{1} \nu_{2}+2 \mu_{2} \nu_{1}\right) \\
& -m\left(\mu_{1} \mu_{2}+\nu_{1} \nu_{2}+\mu_{1} \nu_{2}+\mu_{2} \nu_{1}\right) \\
& -m^{2}\left(\mu_{1}+\mu_{2}+\nu_{1}+\nu_{2}\right)+2 m-2 \alpha(m-1)
\end{aligned}
$$

Mathematische Annalen. XIX. 
(5) $\quad\left(e_{1} f_{1} f_{2} f_{3}\right)=m^{2} \cdot \mu \nu \nu-\alpha m \mu-m \mu-\mu \nu-m^{2}+4 m$ $-\delta_{1}(m \nu \nu-3 \alpha-5)$.

(6) $\left(g_{12} f_{1} f_{2} f_{3}\right)=m^{2}(\mu \mu \nu+\mu \nu \nu-\mu-\nu)-m(\mu \mu+\nu \nu+\mu \nu)$ $-(\alpha-2) m(\mu+\nu)+2 m^{2}-2 m+8 \alpha$.

Aus (5) und (6) folgt:

$$
\begin{aligned}
\left(f_{1} f_{1} f_{3} f_{1}\right) & =m^{2}(\mu \mu \nu \nu-\mu-\nu+4)-\alpha(m+1)(\mu \mu+\nu \nu) \\
& -m(\mu \nu-4 \mu-4 \nu+10)+6 \alpha^{2}+18 \alpha .
\end{aligned}
$$

Fïr $m=1$ ergiebt sich hieraus die in (6) \& 3. borechnete Zah. December 1881. 